\title{
HARDWARE-AWARE COMMUNICATION PROTOCOLS IN LOW ENERGY WIRELESS SENSOR NETWORKS
}

\author{
Fanchun $\mathrm{Jin}^{1}$, Hyeong-Ah $\mathrm{Choi}^{1}$, and Suresh Subramaniam ${ }^{2}$ \\ ${ }^{1}$ Department of Computer Science \\ ${ }^{2}$ Department of Electrical \& Computer Engineering \\ The George Washington University \\ Washington, DC 20052 \\ email:\{jinfc,hchoi,suresh\}@gwu.edu
}

\begin{abstract}
As the energy consumption in sensor nodes is dominated by the radio transmission/reception circuitry, communication protocols must be designed for economy in radio communications. Sensor nodes are generally equipped with short-range radios that have various characteristics including data rate, power consumption in transmit, receive, and sleep modes, and time to switch from one mode to another. These parameters can have significant effects on the performance of communication protocols in low energy sensor networks. In this paper, we consider a protocol called STEM that was proposed in [6] and optimize the protocol's parameters by considering the radio characteristics.
\end{abstract}

\section{INTRODUCTION}

Wireless sensor networks are ad hoc networks composed of a large number of small sensor nodes and one or more "base stations" which process the data received from the sensor nodes and take necessary control actions. Such networks are typically used for monitoring and surveillance functions. A sensor node collects sensory data from its environment, and such data are processed by the node circuitry to determine if an "event" has occurred. Once an event occurs, the node needs to transmit some data collected from the event to a base station. The data transmission phase involves sending the data through other sensor nodes that act as intermediate nodes in a multi-hop network.

The sensor nodes in wireless sensor networks have a limited energy supply that usually cannot be renewed. Thus, the lifetime of a network is constrained by the amount of energy that is spent by the sensor nodes in performing their operation of sensing, processing, and transmitting data to the control centers. Furthermore, the power consumption in sensor nodes is dominated by the radio transmission/reception circuitry. Hence, the communication protocols must be designed such that the energy consumed by the radio circuitry is small as possible. There has been much research on energy-efficient protocols recently [2], [3], [6].

In this paper, we focus on a protocol proposed in [6] called STEM (Sparse Topology Energy Management). The protocol in [6] works as follows. Each node is assumed to be equipped with two radio transceivers - one for data transfer and one for control. Let us suppose that a node $\mathrm{A}$ has sensed an event and wants to transfer some data to node B (which is an intermediate node on the path to a base station). Prior to the data transfer, the link between A and B must be established, as shown in Figure 1. In order to conserve energy during the period when a node is only in the monitoring state and does not have any data to transmit or receive, the STEM protocol does the following. The data radio is in sleep mode until a link to a neighboring node is activated through a control message exchange between the node and one of its neighbors. The control radio of a node sleeps most of the time and wakes up periodically in order to listen to possible requests from neighboring nodes which want to transfer some data to the node. STEM has two versions called STEM-B and STEM-T, where B stands for beacon and $\mathrm{T}$ for tone. In this paper, we will restrict ourselves to STEM-B, and refer to it simply as STEM. Node A transmits a series of beacons (control packets containing A's and B's MAC addresses) to node $\mathrm{B}$ until one of the beacons is received by node $\mathrm{B}$, which then acknowledges the receipt of this packet and turns on its data radio. Upon receiving the acknowledgement from B, A turns on its data radio and the 
link between A and B is activated for data transfer. The duration of each beacon, the inter-beacon interval, the duration of each listen period for B's control radio and the sleep interval for node B's control radio are the protocol's parameters. Note that beacons or acknowledgements may collide with other beacon and/or acknowledgement transmissions, and the protocol ensures that these situations are handled correctly.

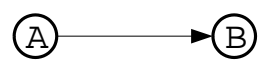

Fig. 1. Node A wants to wake up node B.

While [6] presented an analysis of the energy savings that STEM could offer over a protocol that did not separate the data transfer phase and the monitoring phase, it was not the objective of [6] to optimize the protocol's parameters. Furthermore, some of the characteristics of sensor node radios (such as the time taken for a transceiver to transition from transmit to receive mode) were not taken into account in analyzing the energy savings and link activation latency (to be defined soon). Our goal in this paper is to consider the hardware characteristics of the radios and optimize the protocol's parameters for energy efficiency.

The rest of the paper is organized as follows. The system model, some notation, and the problem we propose to solve are presented in the next section. Results leading to the optimal parameter settings are presented in the subsequent section. This is followed by some numerical results, and then some concluding remarks are made.

\section{THE PROBLEM}

We begin this section by defining some terminology used in describing our problem. Please refer to Figure 2 to follow the notation. As mentioned earlier, node A transmits a series of beacons, and node $\mathrm{B}$ alternates between sleep and receive modes, until one of the beacons is captured by node B, upon which B sends an ACK back to A. This completes the link setup process. Note that there is no synchronization assumed between node $\mathrm{A}$ and node $\mathrm{B}$.

The duration of each beacon is denoted by $T_{T x}$, and its value is determined by the length of each control packet and the data rate of the control radio. Hence, $T_{T x}$ is assumed to be a given parameter in our problem. The time taken for a transceiver to transition from receive to transmit (and from transmit to receive) mode is denoted by $T_{R x-T x}\left(\right.$ and $\left.T_{T x-R x}\right)$. Let $T_{T x}^{\prime}=T_{R x-T x}+T_{T x}+$ $T_{T x-R x}$. Let $T_{T}$ be defined to be the duration of one complete cycle of the transceiver at $A$, i.e., the period from the time when the transceiver starts to transition from receive to transmit mode to the time it starts the next transition to transmit mode.

Similarly, at node $B$, the time taken for a transceiver to transition from sleep to receive (and from receive to sleep) mode is denoted by $T_{S l-R x}$ (and $T_{R x-S l}$ ). The wakeup (i.e., receive) duration of the transceiver is denoted by $T_{R x}$, and we define $T_{R x}^{\prime}=T_{S l-R x}+T_{R x}+T_{R x-S l}$. Let $T_{R}$ denote the period of node B's cycle, i.e., the duration between B's transition from sleep to receive mode and the next such transition.

Typically, there are constraints on the delay for activating the link, and link activation delay can be traded off against energy consumption. The general objective of link activation protocols is to maximize the lifetime of the network subject to satisfying the delay constraints. While this problem may be formulated in a variety of ways, we choose to formulate it as follows.

Let us suppose $I$ is the average interval between requests to set up a link between $\mathrm{A}$ and $\mathrm{B}$, i.e., the period between successive data transfers from A to B. Then, we wish to minimize the energy consumed in an average interval $I$, subject to the link activation delay constraints. Note that node A may also have to receive data from other nodes, and therefore, when it is not sending beacons, it must be following the sleep-receive-sleep cycle as node B. Thus, when A is ready to start sending beacons, its control transceiver may either be in receive or sleep mode. For simplicity, we will assume that it is in receive mode.

Now, the link activation delay, denoted by $D$, is defined to be the period starting from when the transceiver at node $A$ starts to transition from receive to transmit mode for the first beacon to the time when it starts to transition from receive to transmit mode for the last beacon (i.e., the beacon that is captured at $B$ ). If the first beacon is successfully captured, $D=0$. Clearly, $D$ must be an integer multiple of $T_{T}$ and must satisfy the inequality $D \leq D_{0}$, where $D_{0}$ is a given parameter denoting the maximum allowed delay. Let the average value of $D$ be denoted by $D_{\text {ave }}$.

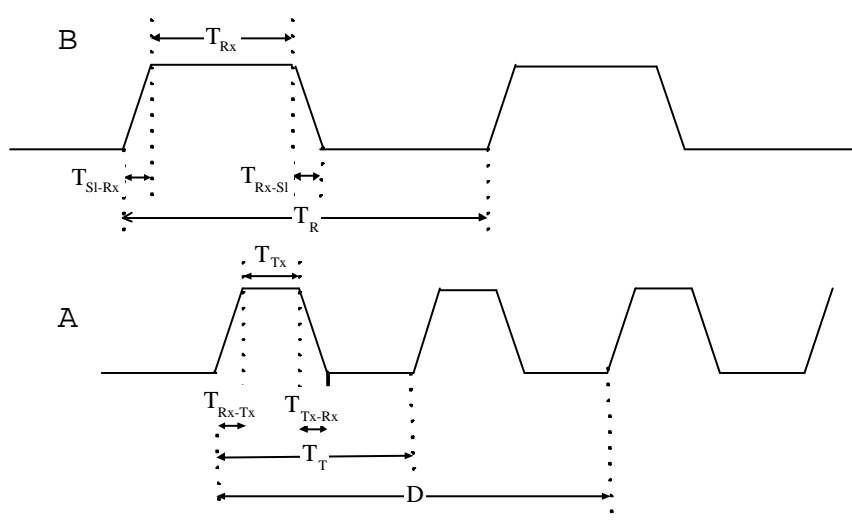

Fig. 2. The timing diagram for A and B's transceivers, and notation. 
Let the transceiver power consumption while it is in transmit, receive, and sleep modes be denoted by $P_{T x}$, $P_{R x}$, and $P_{S l}$, respectively. Let the energy consumed at $A$ during transition periods from receive to transmit mode and from transmit to receive mode together, in one beacon transmit cycle (i.e., within $T_{T}$ ) be denoted by $E_{T x}$. Similarly, let the energy consumed at $B$ during transition periods from sleep to receive mode and from receive to sleep mode together in one wakeup cycle (i.e., within a period $T_{R}$ ) be denoted by $E_{R x}$.

Among the above parameters, $T_{T}, T_{R x}$, and $T_{R}$ are design parameters (i.e., to be determined in our optimization). The remaining are system- or application-specific given values.

Let $E(A)$ and $E(B)$ denote the average amount of energy consumed at nodes $A$ and $B$, respectively, during a period $I$. We then note that

$$
E(A)=\left(T_{T x} P_{T x}+\left(T_{T}-T_{T x}^{\prime}\right) P_{R x}+E_{T x}\right) \cdot D_{a v e} / T_{T}
$$

and

$$
E(B)=\left(T_{R x} P_{R x}+\left(T_{R}-T_{R x}^{\prime}\right) P_{S l}+E_{R x}\right) \cdot I / T_{R} .
$$

Now, the overall energy consumed by the control radios at nodes $A$ and $B$ during the period of $I$ is given by

$$
E=E(A)+E(B) .
$$

We thus have the following problem:

Given parameters as defi ned before, determine values of $T_{T}, T_{R_{x}}$, and $T_{R}$ to minimize $E$ satisfying the condition that the resulting link activation delay $D \leq D_{0}$.

\section{The Optimum Solution}

In this section, we first prove the following result that will be used for setting the optimal parameters.

Lemma 1: For any fixed value of $T_{T}\left(>T_{T x}^{\prime}\right)$, the minimum value of $E(B)$ is achieved when $T_{R x}=T_{T}+T_{T x}$ and $T_{R}=D_{0}$.

Proof: If $T_{R x}$ is less than $T_{T_{x}}$, no beacon from $A$ can be captured by the receiver at $B$; hence, we assume that $T_{R x} \geq T_{T x}$. We consider the following two cases.

Case 1. $T_{R x} \geq T_{T}+T_{T x}$ :

If the transition for the first beacon starts when the transceiver at $B$ is in receive mode (i.e., during its wakeup period) but the remaining time in this wakeup period is less than $T_{T x}+T_{R x-T x}$, the first beacon cannot be captured, but one of the subsequent beacons will be captured during the next wakeup period. Hence, the link activation delay $D$ is bounded by $D<T_{R x-T x}+T_{T x}+T_{R}-$
$\left(T_{R x}+T_{R x-T x}\right)+T_{T}$. As $D \leq D_{0}$, this implies that $T_{R} \leq D_{0}+T_{R x}-T_{T}-T_{T x}$.

Since $P_{R x}>P_{S l}$, for any fixed $T_{R}, E(B)$ is smaller when $T_{R x}=T_{T}+T_{T x}$ than when $T_{R x}>T_{T}+T_{T x}$. Furthermore, when $T_{R x}=T_{T}+T_{T x}$ (i.e., $T_{R} \leq D_{0}$ ), $E(B)$ is smaller when $T_{R}=D_{0}$ than when $T_{R}<D_{0}$. Therefore, the minimum value of $E(B)$ is achieved when $T_{R x}=T_{T}+T_{T x}$ and $T_{R}=D_{0}$.

Case 2: $T_{T x} \leq T_{R x}<T_{T}+T_{T x}$ :

Let $T_{R x}=T_{T x}+z$, where $0 \leq z<T_{T}$. Suppose a beacon is captured within the $k$ th wake-up period of the transceiver at $B$, where the first beacon starts before the 1 st wake-up period. In what follows, we will prove that $z \geq T_{T} / k$. Please refer to Figure 3 .

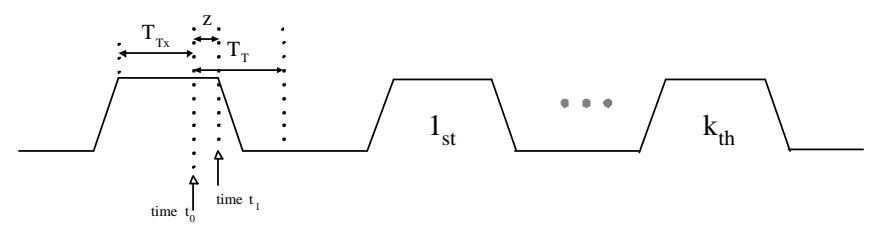

Fig. 3. Beacon captured during the $k$ th wake-up period.

Suppose the first beacon starts between time $t_{0}$ and time $t_{1}$. If the first beacon is captured during the 1st wake-up period, the range of the start time (i.e., time to start to transmit after completing transition from receive to transmit mode) of the first beacon must be bounded by $z$. Similarly, the range of the start time of the first beacon captured by the $i$ th $(1<i \leq k)$ wake-up period is bounded by $z$. To guarantee the first beacon captured by the $k$ th wake-up period, $k z \geq T_{T}$, which implies $z \geq T_{T} / k$.

If a beacon is captured during the $k$ th wake-up period, the link activation delay is $D=k T_{R}-z$ in the worst case. As $D \leq D_{0}$, it implies that $k T_{R}-z \leq D_{0}$. As $z \geq T_{T} / k$ (i.e., $k \geq T_{T} / z$ ), we have $D_{0} \geq \frac{T_{T} T_{R}}{z}-z$.

Note that the minimum value of $E(B)$ in Case 1 is achieved when $T_{R x}=T_{T x}+T_{T}$ and $T_{R}=D_{0}$. In Case 2, a lower bound of $E(B)$ is achieved by substituting $T_{R}$ by $\left(D_{0}+z\right) z / T_{T}$ (as $\left.T_{R} \leq\left(D_{0}+z\right) z / T_{T}\right)$ and $T_{R x}=T_{T x}+z$. It is then seen that the minimum value of $E(B)$ in Case 1 is no larger than that in Case 2. This completes the proof of the lemma.

Note that $T_{T}$ is the only design parameter in $E(A)$, and hence, $E$ is optimized for a given $T_{T}$ by setting $T_{R x}=$ $T_{T}+T_{T x}$ and $T_{R}=D_{0}$ according to Lemma 1. Also, we need to find $D_{\text {ave }}$ in order to determine $E(A)$ and, thence, find the optimal value of $T_{T}$ that minimizes $E$. We proceed to do that now.

For purposes of explanation, let us say that a starting point of a beacon is the instant of time at which A's transceiver has just finished its transition from receive to 
transmit mode, and the starting point of a wakeup period is the instant of time at which B's transceiver has just finished its transition from sleep to receive mode. According to Lemma 1, in an optimal solution, $T_{R x}=T_{T}+T_{T x}$. If this is the case, then the beacon is captured in either the wakeup period during which beacon transmission starts, or the next wakeup period. Because of this fact, we only need to consider the case that the beacon transmission starts anywhere over a period of $T_{R}$ in order to determine the average delay.

Now, if beacon transmission starts within a time interval $T_{R x}-T_{T x}$ from the starting point of a wakeup period, then the delay is 0 . If beacon transmission starts within a period $T_{T}$ prior to the starting point of a wakeup period, then the delay is $T_{T}$. If it starts within period $T_{T}$ prior to the above interval, then the delay is $2 T_{T}$ and so on. As A's and B's transceivers are completely asynchronous, the beacon transmission starting time is uniformly distributed over the interval $T_{R}$. Since $\frac{T_{R}-\left(T_{R x}-T_{T x}\right)}{T_{T}}$ is not necessarily an integer, we have the following expression for the average delay.

$$
\text { Let } \beta=\left\lfloor\frac{T_{R}-\left(T_{R x}-T_{T x}\right)}{T_{T}}\right\rfloor \text {. }
$$

Then,

$$
\begin{aligned}
D_{a v e}= & \frac{T_{T}}{T_{R}} T_{T}(1+2+\cdots+\beta)+ \\
& \frac{T_{R}-\left(T_{R x}-T_{T x}\right)-\beta T_{T}}{T_{R}}(\beta+1) T_{T} \\
= & \frac{T_{T}}{T_{R}}\left[\left[T_{R}-\left(T_{R x}-T_{T x}\right)\right](\beta+1)-\right. \\
& \left.\frac{\beta(\beta+1)}{2} T_{T}\right] .
\end{aligned}
$$

Now, $E(A)$ can be obtained by substituting for $D_{\text {ave }}$ in (1). Unfortunately, because of the floor function in $\beta$, it is not possible to get an analytical expression for the optimal value of $T_{T}$ that minimizes $E$, and the optimal value of $T_{T}$ has to be determined numerically. In the next section, we present some performance evaluation results.

\section{EXPERIMENTAL RESULTS}

We assume that the control radios are the TR1000 radio transceivers [1]. We assume that the data rate for the radio is $2.4 \mathrm{kbps}$, the size of beacon is $144 \mathrm{bits}$, and the size of acknowledgment is 144 bits (following [6]). For this data rate, the parameters of the TR1000 radio are: $P_{T x}=36$ $\mathrm{mW}, P_{R x}=5.4 \mathrm{~mW}, P_{S l}=2.1 \mu \mathrm{W}, T_{T x-R x}=19.2 \mathrm{~ms}$, $T_{R x-T x}=12 \mu \mathrm{s}, T_{S l-R x}=19.2 \mathrm{~ms}$, and $T_{R x-S l}=10$ $\mu \mathrm{s}$.

We can calculate $T_{\mathrm{Tx}}$ and $\alpha_{\mathrm{T}}$, the minimum possible value of $\left(T_{T}-T_{T x}\right)$ using the following equation.
- $T_{\mathrm{Tx}}=$ BeaconSize/DataRate

- $\alpha_{\mathrm{T}}=$ AcknowledgmentSize/DataRate + $T_{T x-R x}+T_{R x-T x}$.

Details about the power consumption during the transition periods from one mode to another could not be found for the TR1000, and therefore, we present results for three assumptions regarding the power consumption as given below.

Assumption 1:

When changing to mode $\mathrm{Y}$ from mode $\mathrm{X}$, the power consumption is the power consumption at mode $\mathrm{Y}$ during the mode transition period. Thus,

$$
E_{T x}=P_{T x} T_{R x-T x}+P_{R x} T_{T x-R x},
$$

and

$$
E_{R x}=P_{R x} T_{S l-R x}+P_{S l} T_{R x-S l} .
$$

Assumption 2:

When changing to mode $\mathrm{Y}$ from mode $\mathrm{X}$, the power consumption is the power consumption at mode $\mathrm{X}$ during the mode transition period. Thus,

$$
E_{T x}=P_{R x} T_{R x-T x}+P_{T x} T_{T x-R x},
$$

and

$$
E_{R x}=P_{S l} T_{S l-R x}+P_{R x} T_{R x-S l} .
$$

Assumption 3:

When changing to mode $\mathrm{Y}$ from mode $\mathrm{X}$, the power consumption is the average of power consumption at modes $\mathrm{X}$ and $\mathrm{Y}$, during the mode transition period. Thus,

$$
E_{T x}=\frac{\left(P_{R x}+P_{T x}\right)\left(T_{R x-T x}+T_{T x-R x}\right)}{2},
$$

and

$$
E_{R x}=\frac{\left(P_{R x}+P_{S l}\right)\left(T_{R x-S l}+T_{S l-R x}\right)}{2} .
$$

Then, we can get the following data from the assumptions above and the specifications.

TABLE I

SYSTEM PARAMETERS

\begin{tabular}{|l|l|l|l|}
\hline & Ass. 1 & Ass. 2 & Ass. 3 \\
\hline$T_{\mathrm{Tx}}(m s)$ & 60 & 60 & 60 \\
\hline$\alpha_{\mathrm{T}}(m s)$ & 79.212 & 79.212 & 79.212 \\
\hline$E_{\mathrm{Tx}}(\mu J)$ & 104.11 & 691.26 & 397.69 \\
\hline$E_{\mathrm{Rx}}(\mu J)$ & 103.68 & 0.09 & 51.89 \\
\hline
\end{tabular}

Throughout this section, we assume $D_{0}=1$ minute, unless otherwise specified. We first plot $E$ as a function 
of $T_{T}$ for $I=5$ hours in Figure 4. Notice that there is an optimal value of $T_{T}$ that is dependent on the assumption that is used regarding the power consumption during transceiver mode transitions.

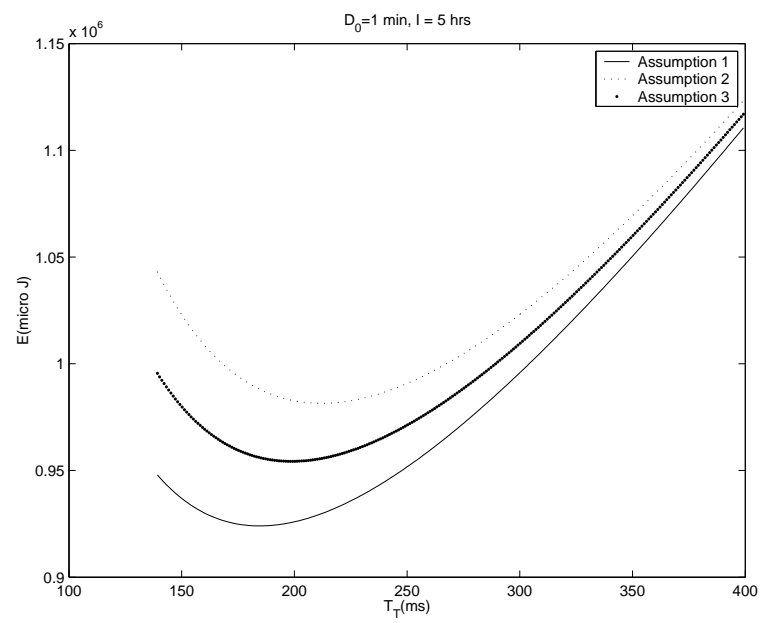

Fig. 4. $E$ vs. $T_{T}$ for $D_{0}=1 \mathrm{~min}$. and $I=5$ hours.

We next plot the optimal value of $T_{T}$ (obtained numerically) against $I$ in Figure 5. We observe that $T_{T}$ decreases monotonically with $I$. This can be explained by the fact that $E(B)$ increases with $I$ and this increase can be compensated by decreasing $T_{R x}\left(=T_{T}+T_{T x}\right.$ for optimality). However, notice that $T_{T}$ is lower bounded by $T_{T x}+\alpha_{T}$ $=139.212$ for the parameters in Table I, and therefore, the optimal $T_{T}$ for large values of $I$ can be expected to be equal to this lower bound.

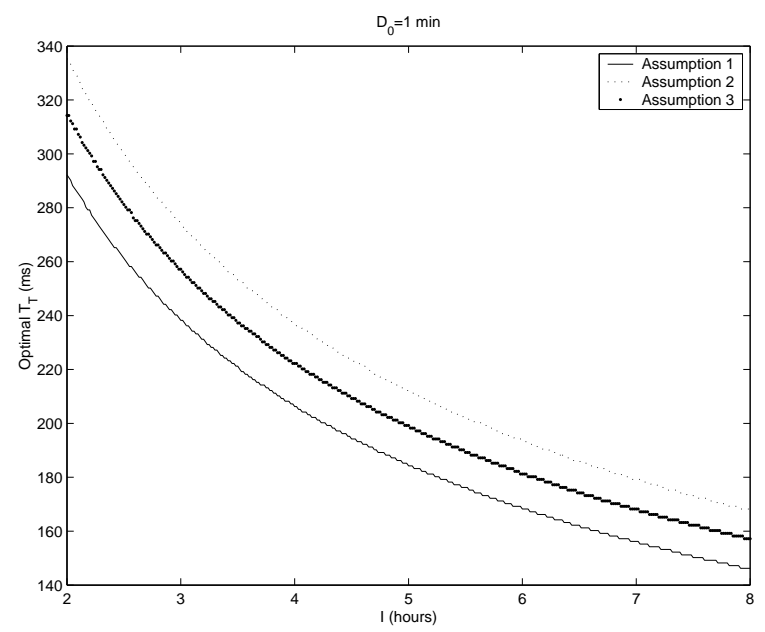

Fig. 5. Relationship between $T_{\mathrm{T}}$ and $I$.

In Figure 6, we fix $I$ at 1 day, and plot the optimal value of $T_{T}$ against the link activation delay bound $D_{0}$. As explained above, the optimal value of $T_{T}$ is equal to the lower bound initially. However, as $D_{0}$ increases, the optimal value of $T_{T}$ increases beyond this lower bound.

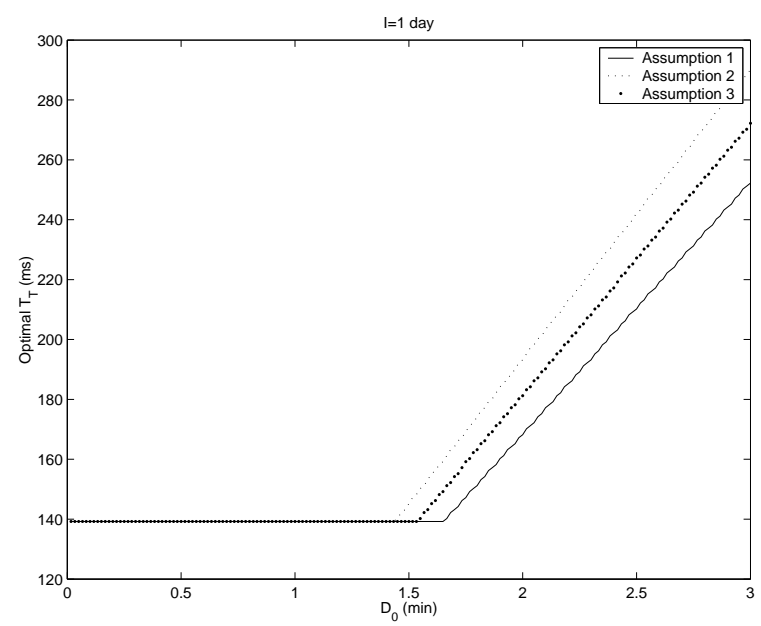

Fig. 6. Optimal $T_{T}$ vs. $D_{0}$ for $I=1$ day.

Next, in Figure 7, the lifetime of a node is plotted as a function of $T_{T}$ for $I=1$ hour. Here, the total energy of each node, $\mathcal{E}$, is assumed to be 75 Joules, and it is assumed that each node requests data transfer at intervals of $I$ for $50 \%$ of its lifetime and is in the sleep/wakeup cycle listening for data transfer requests the other $50 \%$ of its lifetime. Hence, lifetime is given by $2 \mathcal{E} I / E$. Notice that the lifetime can vary by up to $50 \%$ depending on the value of $T_{T}$ that is chosen, in Figure 7.

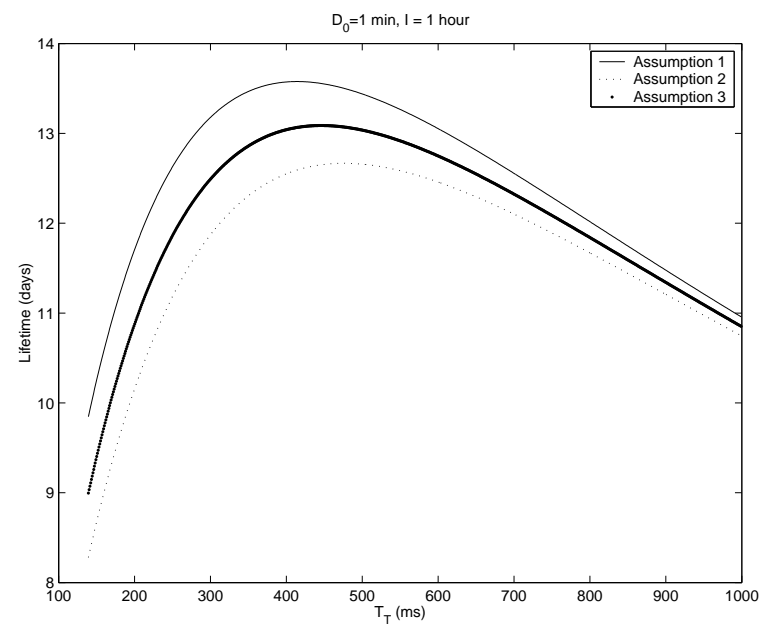

Fig. 7. Node lifetime vs. $T_{T}$ for $I=1$ hour.

Choosing $T_{T}$ appropriately is even more important when $I$ is large ( 1 day) as Figure 8 shows. Here, the lifetime continuously decreases from about 62 days as $T_{T}$ increases.

Finally, we plot the node's lifetime (with optimal parameters) as $I$ increases in Figure 9. Here, we see that the lifetime increases rapidly initially and then gradually flattens out. This is because, for large $I, T_{T}$ reaches its lower bound and thus all design parameters are fixed. 


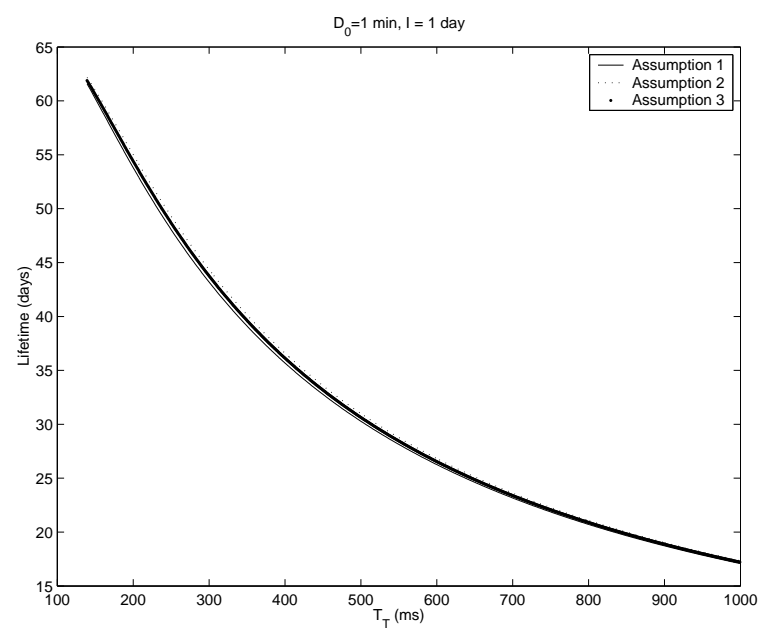

Fig. 8. Node lifetime vs. $T_{T}$ for $I=1$ day.

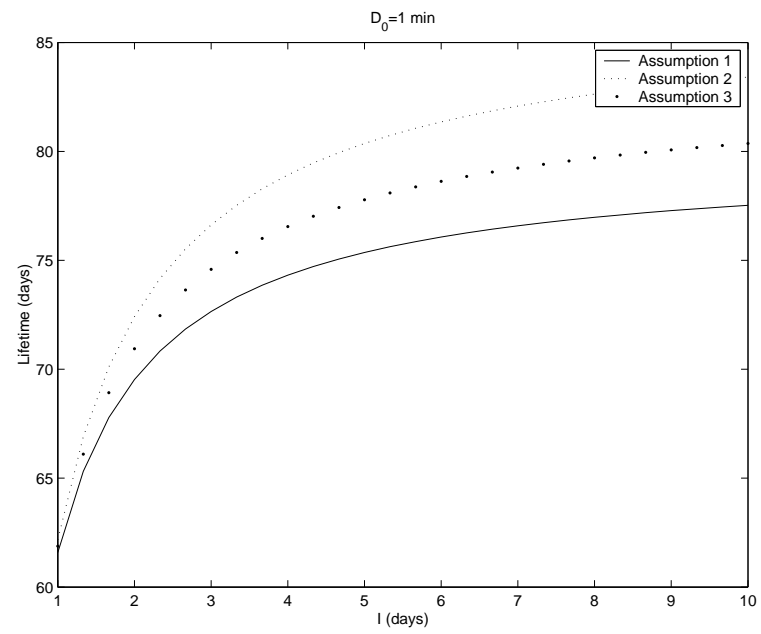

Fig. 9. Optimal node lifetime vs. $I$.

\section{CONCLUDING REMARKS}

Wireless sensor networks are a topic of intensive research these days because of their wide range of applications and the decreasing costs of sensors and short range radio transceivers. An important consideration in designing communication protocols for sensor networks is the energy consumption. In this paper, we considered a protocol for sensor networks called STEM and focused on optimizing its parameters. The optimization considered such radio characteristics as the time to transition from one mode to another and the power consumption during these times. Results show the performance of a node can vary significantly depending on the system and protocol parameters.

\section{Acknowledgement}

This work was supported in part by U.S. Army Research Office under contract DAAD 19-02-D-001,
TCN02-007.

\section{REFERENCES}

[1] RF Monolithics Inc., http://www.rfm.com, ASH Transceiver TR1000 Data Sheet

[2] W. Ye, J. Heidemann, and D. Estrin, "An Energy-Effi cient MAC Protocol for Wirelss Sensor Networks,"INFOCOM, 2002, pp.1567-1576.

[3] W. Ye, H. Heidemann, and D. Estrin, 'Medium Access Control with Coordinated, Adaptive, Sleeping for Wirelss Sensor Networks,”UCI/ISI Technical Report, ISI-TR-567, January 2003.

[4] C. S. Raghavendra and S. Singh, 'PAMAS - Power Aware MultiAccess Protocol with Signalling for Ad Hoc Networks," Computer Communications Review, 1998.

[5] B. Chen, K. Jamieson, H. Balakrishnan, and R. Moris, 'Span: an energy effi cient coordination algorithms for topology maintenance in ad hoc wirelss networks," MobiCom, 2001, pp. 85-96.

[6] C. Schurgers, C. Tsiatsis, S. Ganeriwal, and M. Srivastava, 'Optimizing Sensor Networks in the Energy-Latency-Density Design Space," IEEE Trans. On Mobile Computing, Vol. 1, No. 1, January-March 2002, pp. 70-80.

[7] A. Y. Wang, S. Cho, C. G. Sodini, and A. Chandrakasan, 'Energy Effi cient Modulation and MAC for Aymmetric RF Microsensor Systems," Proc. International Symposium on Low Power Electronics and Design (ISLPED), 2002, pp.106-111. 